\title{
Perception and Recognition Memory in Monkeys Following Lesions of Area TE and Perirhinal Cortex
}

\author{
Elizabeth A. Buffalo, ${ }^{2}$ Seth J. Ramus, ${ }^{4}$ Larry R. Squire,,${ }^{1,2,3}$ and Stuart M. Zola ${ }^{1,2,3,5}$ \\ ${ }^{1}$ Veterans Affairs San Diego Healthcare System, San Diego, California 92161 USA; Departments of ${ }^{2}$ Neurosciences and ${ }^{3}$ Psychiatry, UCSD, La \\ Jolla, California 92093, USA; ${ }^{4}$ Department of Psychology, Boston University, Boston, Massachusetts 02134, USA.
}

\begin{abstract}
Monkeys with lesions of perirhinal cortex (PR group) and monkeys with lesions of inferotemporal cortical area TE (TE group) were tested on a modified version of the delayed nonmatching to sample (DNMS) task that included very short delay intervals $(0.5 \mathrm{sec})$ as well as longer delay intervals ( $1 \mathrm{~min}$ and $10 \mathrm{~min})$. Lesions of the perirhinal cortex and lesions of area TE produced different patterns of impairment. The PR group learned the DNMS task as quickly as normal monkeys $(\mathrm{N})$ when the delay between sample and choice was very short $(0.5 \mathrm{sec})$. However, performance of the PR group, unlike that of the $\mathrm{N}$ group, fell to chance levels when the delay between sample and choice was lengthened to $10 \mathrm{~min}$. In contrast to the PR group, the TE group was markedly impaired on the DNMS task even at the 0.5-sec delay, and three of four monkeys with TE lesions failed to acquire the task. The results provide support for the idea that perirhinal cortex is important not for perceptual processing, but for the formation and maintenance of long-term memory. Area TE is important for the perceptual processing of visual stimuli.
\end{abstract}

Recent findings demonstrated a double dissociation between the effects of two adjacent temporal lobe regions, the perirhinal cortex (PR) and inferotemporal cortical area TE (Buffalo et al. 1999). The pattern of findings suggested that the perirhinal cortex, like other medial temporal lobe structures, is important for the formation of memory and for retaining information across delays. By contrast, area TE is important for visual perceptual processing.

In one experiment (Experiment 1, in Buffalo et al. 1999), the monkeys with lesions of the PR and the monkeys with lesions of area TE were comparably impaired on the delayed nonmatching to sample task (DNMS) when it was administered in the visual modality. Specifically, both operated groups were impaired at the initial learning of the task when the delay interval between sample presentation and the choice phase was $8 \mathrm{sec}$. Both groups also were impaired when the delay interval was extended to $15 \mathrm{sec}, 1 \mathrm{~min}, 10$ $\mathrm{min}$, and $40 \mathrm{~min}$. With respect to initial learning, the finding that the monkeys with TE lesions were impaired was consistent with the idea that area TE is important for visual perceptual processing. However, the impaired performance of the PR monkeys during initial learning was less easy to understand, because an 8-sec delay seems too short to place much demand on memory.

At least two possibilities can be considered to account

${ }^{5}$ Corresponding author.

E-MAIL szola@ucsd.edu; FAX (858) 534-1569.

Article and publication are at www.learnmem.org/cgi/doi/10.1101/ $\operatorname{lm} 32100$. for the impaired performance of the PR monkeys. First, the PR monkeys, like the monkeys with TE lesions, may have had some impairment in perceptual processing that affected their performance at the 8-sec delay. The idea that PR damage impairs perceptual processing or object identification has been suggested previously (Eacott et al. 1994; Buckley and Gaffan 1997; Murray and Bussey 1999). A second possibility is that a delay of 8 sec may have been long enough to detect a delay-dependent memory impairment. Indeed, amnesic patients with perirhinal damage performed well on a visual recognition memory test at delays of 0 to 2 sec, but were impaired at delays of 6 to $10 \mathrm{sec}$ (Buffalo et al. 1998a).

To test these two possibilities, we trained monkeys on the DNMS task using a very short delay $(0.5 \mathrm{sec})$ between the presentation of the sample and choice stimuli and we then compared the effects of PR and TE lesions on performance. Our predictions follow from findings in monkeys and humans with medial temporal lobe damage that immediate or short-term memory is intact and long-term memory is impaired (Overman et al. 1990; Alvarez-Royo et al. 1992; Buffalo et al. 1998a; Alvarez et al. 1994). If the PR is involved primarily in memory, monkeys with lesions limited to the PR should be able to perform the DNMS task normally at very short delays, that is, when the memory requirement of the task is minimal. Alternatively, if the PR is involved in visual perceptual processing or in object identification, lesions of the PR should impair performance on the DNMS task, even at very short delays. Likewise, if area TE is im-

LEARNING \& MEMORY 7:375-382 @ 2000 by Cold Spring Harbor Laboratory Press ISSN1072-0502/00 \$5.00

$$
\begin{aligned}
& \begin{array}{llllllllllllllllllllllllllll}
L & E & A & R & N & I & N & G & \bigotimes & M & E & M & O & R & Y
\end{array} \\
& \text { www.learnmem.org }
\end{aligned}
$$


portant for visual perceptual processing, lesions of area TE should severely impair performance on the DNMS task, even at very short delays.

\section{RESULTS}

\section{Histological Results}

\section{Description of the Lesions in the PR Group}

Each of the lesions in the PR group has been illustrated and described in detail previously (Buffalo et al. 1999), and only a brief description will be provided here. Figure 1 shows photomicrographs from monkey PR 2, whose lesion most closely approximated the intended lesion. On average, the
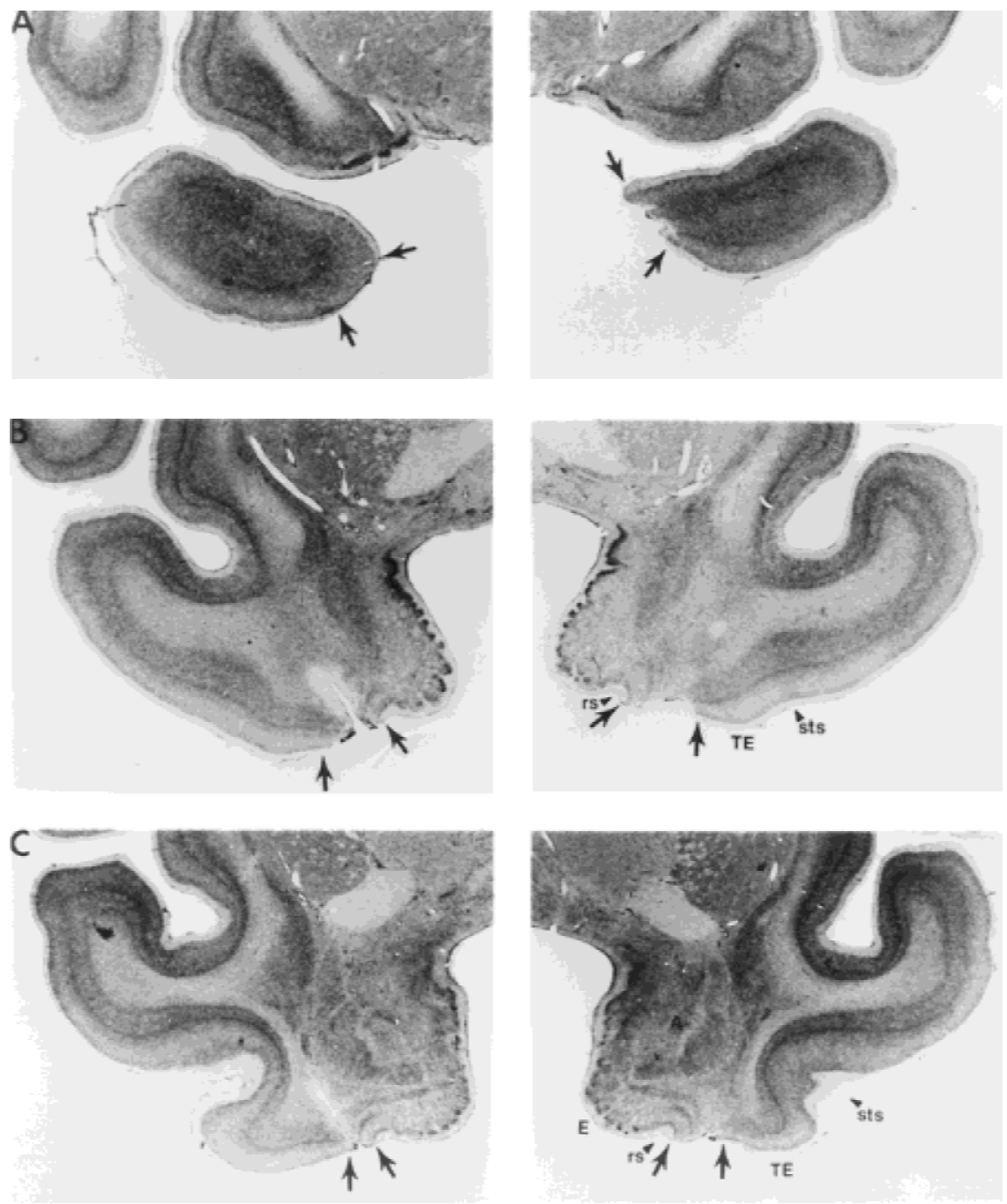

Figure 1 Photomicrographs of representative sections through the left and right temporal lobes of monkey PR 2, whose lesion most closely approximated the intended lesion. The sections are arranged from rostral $(A)$ to caudal $(E)$ (also see facing page), and the lesion is indicated by arrows at each level. rs, rhinal sulcus; sts, superior temporal sulcus; TE, area TE; A, amygdala; $\mathrm{E}$, entorhinal cortex; $\mathrm{H}$, hippocampal region.
PR group sustained an estimated $74 \%$ bilateral damage to the PR (PR $1=73 \%$; PR $2=87 \%$; PR $3=65 \%$; PR $4=69 \%$; PR $5=77 \%$; the average damage without PR 1=74\%). All monkeys sustained some damage to the PR at the polar region, although in most cases the most dorsal part of area 36 was spared at the pole. In all cases, there was extensive damage to the PR beginning rostral to the amygdala and extending through the caudal extent of the PR. In all cases except PR 1, the amount of unintended damage outside the PR was minimal. Monkey PR 1 sustained significant unilateral damage to area TE (see Materials and Methods). In monkeys PR 2-PR 5, there was little or no direct damage to area TE or to rostral parahippocampal cortex. Additionally, in none of the cases was there damage to the amygdala, the entorhinal cortex, or the hippocampal region. There was minimal white matter damage in all cases.

\section{Description of the Lesions in the TE Group}

Each of the lesions in the TE group has been illustrated and described in detail previously (Buffalo et al. 1999). Figure 2 shows photomicrographs from monkey TE 4, whose lesion most closely approximated the intended lesion. On average, the TE group sustained an estimated $62 \%$ bilateral damage to area TE (TE $1=$ $44 \%$; TE $3=69 \%$; TE $4=64 \%$; TE $5=71 \%)$. All monkeys sustained moderate to extensive removal of area TE throughout its rostrocaudal extent. The amount of unintended damage outside area TE was minimal in all cases, and there was little or no damage to the PR. However, monkey TE 5 sustained minimal bilateral compression of the PR extending $\sim 2 \mathrm{~mm}$ on the left and $\sim 1 \mathrm{~mm}$ on the right. Monkey TE 5 also sustained a minimal amount of damage to the parahippocampal cortex. None of the cases sustained damage to the entorhinal cortex, the amygdala, or the hippocampal region. There was moderate white matter damage in two cases (TE 3 and TE 5).

\section{Behavioral Results}

Figure $3 \mathrm{~A}$ shows the mean number of trials required by each group to reach criterion level performance on the DNMS task during initial learning

$$
\begin{array}{lllllllllllllll}
L & E & A & R & N & I & N & G & \mathcal{Q} & M & E & M & O & R & Y \\
\text { www.learnmem.org } & &
\end{array}
$$



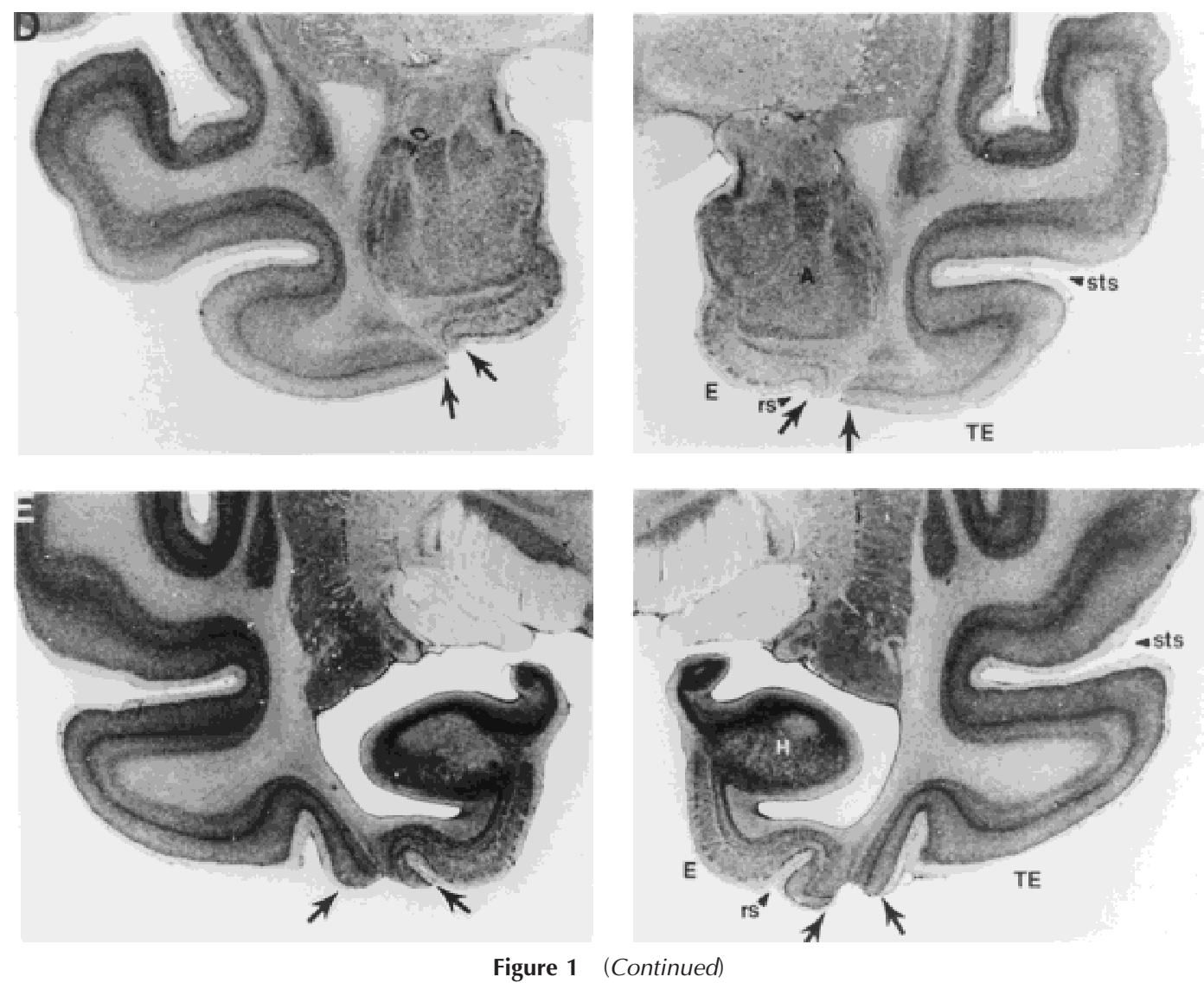

Figure 1 (Continued)

with a delay of $0.5 \mathrm{sec}$ (for performance of individual monkeys, see Table 1). The TE group required more trials to learn the task than did the normal monkeys (N group) and the PR group (Mann-Whitney U test, both Ps <.05, onetailed; $P<.07$, and $P<.08$, respectively, two-tailed; because of the ceiling performance of animals in the TE group, nonparametric statistics were used to analyze the data from this portion of the task). In fact, three of the four monkeys in the TE group were unable to learn the task within 3000 trials. By contrast, the performance of the PR group was not different from that of the $\mathrm{N}$ group $(P>.10)$. When data for monkey PR 1 were included in the analyses, the results were similar: the PR monkeys were unimpaired on the task, and the TE monkeys were numerically impaired relative to the PR monkeys; $P<.08$, one-tailed.

Figure $3 \mathrm{~B}$ and $\mathrm{C}$ show the performance of the monkeys in the PR group and unoperated control monkeys $(\mathrm{N})$ on the delay portion of the delayed nonmatching to sample task (for individual scores, see Table 1). Monkeys TE 1, 3, and 5, and monkey PR 1 were not tested on the delay portion of the task because they did not reach criterion level performance at the 0.5-sec delay. A two-way ANOVA (two groups: PR and N; three delays: $1 \mathrm{sec}, 1 \mathrm{~min}$, and 10 min) revealed a significant effect of delay $[F(2,10)=105.90$,
$P$ s <.01], and no significant effects of group, or group $\mathrm{X}$ delay interaction $(P \mathrm{~s}>.10)$. The scores of the PR group were similar to the scores of the normal group at the 1-sec and 1-min delays. At the 10-min delay, the PR group performed numerically worse than the $\mathrm{N}$ group, but this difference did not reach statistical significance (PR group $=54.5 \%$ correct, $\mathrm{N}$ group $=61.3 \%$ correct, $P>.10)$. The $\mathrm{N}$ group performed significantly above chance $(P<.05)$ at the 10 -min delay, while the performance of the PR group was at chance levels $(P>.10)$.

In summary, at the short delay of $0.5 \mathrm{sec}$, the TE group performed worse than both the $\mathrm{N}$ group and the PR group. By contrast, the PR group performed normally. At the longest delay tested (10 $\mathrm{min}$ ), the PR group performed numerically worse than the $\mathrm{N}$ group, and unlike the $\mathrm{N}$ group, did not perform significantly above chance. These findings demonstrate that PR lesions did not affect performance at very short delays, while lesions of area TE produced a profound deficit at a very short delay.

\section{DISCUSSION}

On an automated version of the visual DNMS task, monkeys with bilateral lesions limited to the PR learned the DNMS task as quickly as normal monkeys when the delay between

$$
\begin{array}{lllllllllllllll}
L & E & A & R & N & I & N & G & \mathcal{Z} & M & E & M & O & R & Y \\
\text { www.learnmem.org } & &
\end{array}
$$



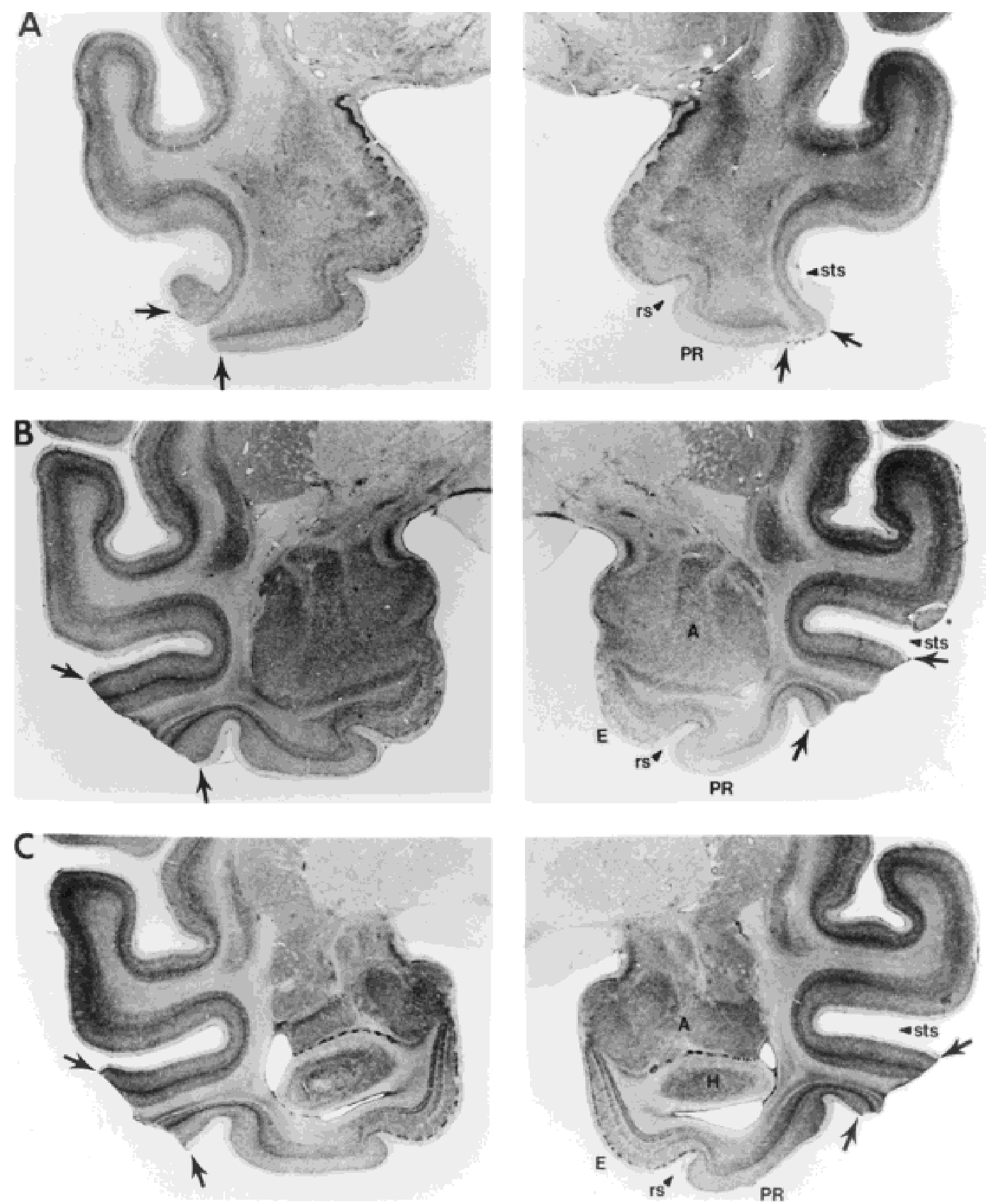

Figure 2 Photomicrographs of representative sections through the left and right temporal lobes of monkey TE 4, whose lesion most closely approximated the intended lesion. The sections are arranged from rostral $(A)$ to caudal $(E)$ (also see next page), and the lesion is indicated by arrows at each level. The asterisk indicates a processing artifact. rs, rhinal sulcus; sts, superior temporal sulcus; PR, perirhinal cortex; A, amygdala; $E$, entorhinal cortex; $H$, hippocampal region; $\mathrm{PH}$, parahippocampal cortex.

sample and choice was very short (0.5 sec; Fig. 3A). However, monkeys in the PR group performed at chance levels when the delay between sample and choice was lengthened to $10 \mathrm{~min}$ (Fig. 3C). In contrast to the PR group, monkeys with bilateral lesions limited to area TE were impaired markedly on the DNMS task even when the delay interval was very short $(0.5 \mathrm{sec})$. In fact, only one monkey in the TE group was able to learn the task within 3000 trials.

Taken together with previous findings with the visual paired-comparison task, another task of recognition memory (Buffalo et al. 1999), the present findings point to a dissociation between the effects of PR lesions and area TE lesions

$$
\begin{array}{lllllllllllllll}
\text { L } & E & A & R & N & I & N & G & \mathbf{Z} & M & E & M & O & R & Y \\
\text { ww.learnmem.org } & & &
\end{array}
$$



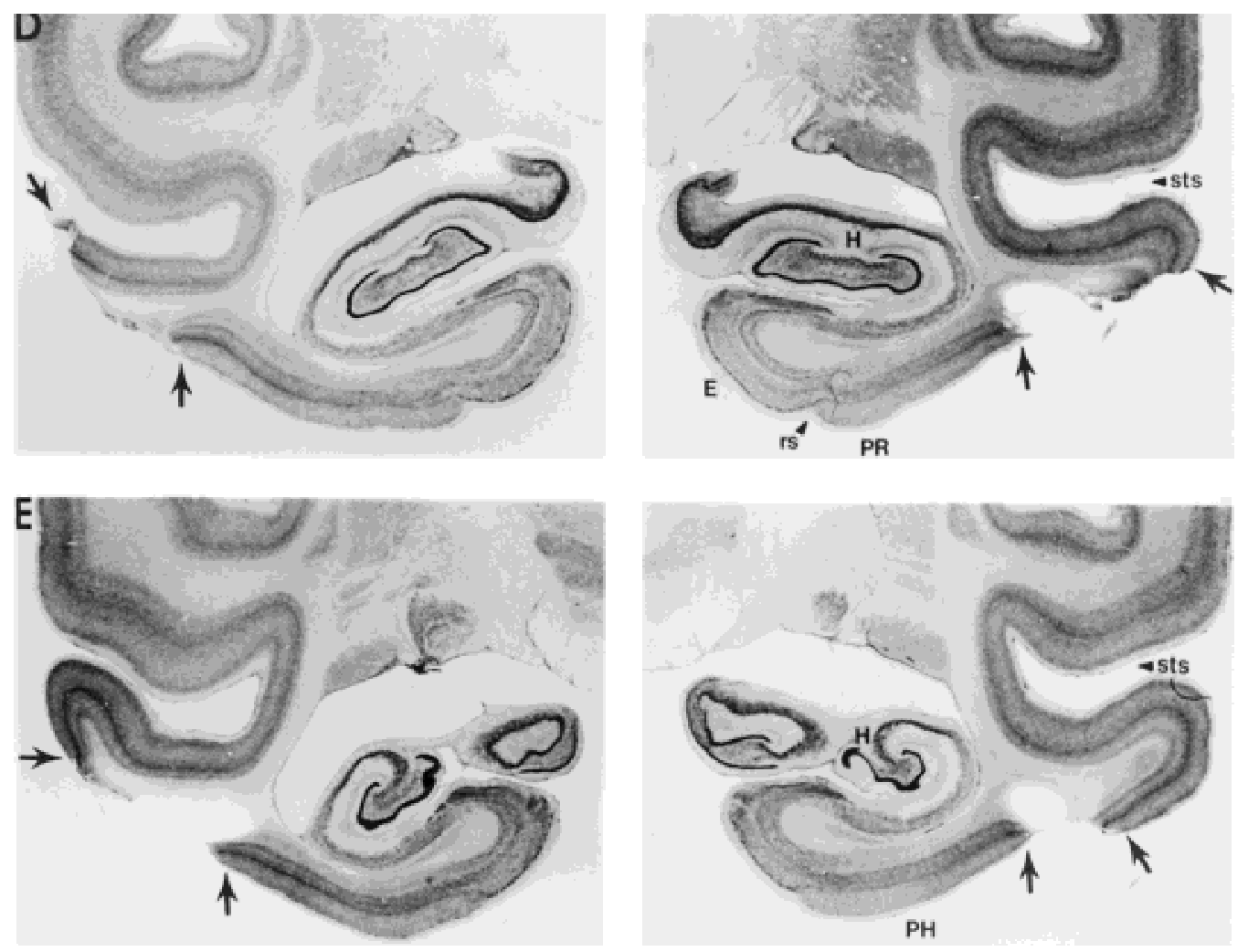

Figure 2

on visual recognition memory performance. On both tasks of recognition memory, lesions of the PR spared immediate memory, i.e., with a delay interval $\leq 1.0 \mathrm{sec}$. In contrast, lesions of area TE severely impaired performance in both tasks, even at delays as short as $0.5 \mathrm{sec}$ and $1 \mathrm{sec}$. The impairment at short delays suggests that the monkeys in the
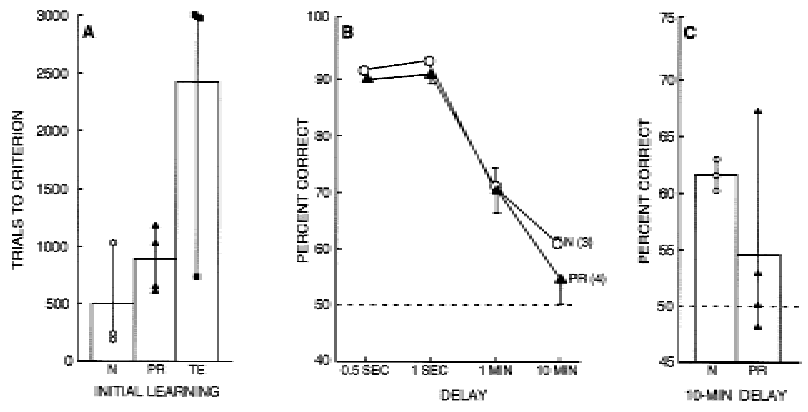

Figure 3 (A) Initial learning of the automated visual delayed nonmatching to sample task (DNMS) at a delay of $0.5 \mathrm{sec}$ by normal monkeys $(\mathrm{N}=3)$, monkeys with lesions of the perirhinal cortex $(P R=4)$, and monkeys with lesions of area TE $(T E=4)$. Symbols show trials to criterion for individual monkeys. (B) Performance across delays for the normal monkeys $(\mathrm{N}=3)$ and the monkeys with lesions of the perirhinal cortex (PR-4). Bars represent standard errors of the mean. (C) An expanded view of the performance of the $\mathrm{N}$ and PR groups at the 10-min delay. Symbols show the performance of individual monkeys.
(Continued)

TE group could not adequately perceive, attend to, or process the visual stimuli. This difficulty is specific to the visual modality, because the same monkeys performed normally on a recognition memory task in the tactual modality (Buffalo et al. 1999).

Other work has led to the proposal that the function of the PR is distinct from the function of other medial temporal lobe structures and that PR is involved in the perceptual processing of visual stimuli (Eacott et al. 1994) or in the identification of individual objects (Buckley and Gaffan 1997). In the case of object identification (Buckley and Gaffan 1997, 1998a,b), lesions of the PR were reported to impair performance only when the perceptual requirements of the task were increased. However, a clear interpretation of these findings is difficult, because the three monkeys that participated in all of these studies sustained inadvertent damage to area TE that appeared sufficient to account for the impaired performance (Buffalo et al. 1998b).

In the case of perceptual processing (Eacott et al. 1994), monkeys with combined lesions of the PR and entorhinal cortex ( $\mathrm{Rh}$ group) were given five separate tasks of delayed matching-to-sample (MTS) that included simultaneous-matching and 0 -sec delay conditions. When performance on these conditions was combined across two of the five tasks (tasks A and D in Eacott et al. 1994), the operated

$$
\begin{array}{lllllllllllllll} 
& E & A & R & N & I & N & G & \mathbf{Z} & M & E & M & O & R & Y \\
\text { www.learnmem.org } & & &
\end{array}
$$


Table 1. Performance on the Delayed Nonmatching to Sample Task

\begin{tabular}{lccccc}
\hline & & \multicolumn{5}{c}{ Delays } \\
\cline { 5 - 6 } Group & $\begin{array}{l}\text { Trials to } \\
\text { criterion }\end{array}$ & $0.5 \mathrm{sec}$ & $1 \mathrm{sec}$ & $1 \mathrm{~min}$ & $10 \mathrm{~min}$ \\
\hline Normal & & & & & \\
1 & 229 & 93 & 94 & 77 & 63 \\
2 & 1057 & 93 & 94 & 72 & 61 \\
3 & 200 & 91 & 94 & 66 & 60 \\
Mean & 495 & 92 & 94 & 72 & 61 \\
PR & & & & & \\
1 & $3000^{*}$ & 71 & - & - & - \\
2 & 668 & 90 & 94 & 78 & 67 \\
3 & 1208 & 90 & 88 & 76 & 48 \\
4 & 1040 & 90 & 90 & 64 & 50 \\
5 & 676 & 92 & 93 & 64 & 53 \\
Mean (PR 2-5) & 898 & 91 & 91 & 71 & 55 \\
TE & & & & & \\
1 & $3000^{*}$ & 77 & - & - & - \\
3 & $3000^{*}$ & 82 & - & - & - \\
4 & $3000^{*}$ & 85 & - & - & - \\
5 & 716 & 92 & 93 & 76 & 72 \\
\hline
\end{tabular}

(PR) Monkeys with bilateral lesions of perirhinal cortex. (TE) Monkeys with bilateral lesions of area TE. $\left(^{*}\right)$ Animal did not reach criterion on this task with a 0.5 -sec delay and was not tested at the longer delays. For these monkeys, the score for the 0.5 -sec delay is the percent correct score that the animal had achieved when training was discontinued. See text for histological findings in the case of monkey PR 1. Monkey TE 2 from Buffalo et al. (1999) did not participate in the present study.

monkeys were impaired at the 0-sec and simultaneous matching conditions. However, this study does not provide strong evidence for an impairment in visual perceptual processing following PR lesions. Specifically, across all five tasks (tasks A, A1, C, D, and E), the operated monkeys performed similarly to, and not significantly different from, the control monkeys on both the simultaneous-matching and the 0-sec delay conditions (all $P$ s $>1.0$; Eacott, personal communication). Moreover, for the two tasks that were most similar to the task used in the present study (tasks A and A1 in Eacott et al. 1994), the operated monkeys also exhibited intact performance on the simultaneous-matching and 0 -sec delay conditions (task A: simultaneous, $\mathrm{Rh}=90 \%$; $\mathrm{N}=94.8 \% ; P>.10 ; 0-\mathrm{sec} ; \mathrm{Rh}=94 \% ; \mathrm{N}=98.3 \% ; P>.10 ;$ task A1: simultaneous, $\mathrm{Rh}=97.3 \% ; \mathrm{N}=97.8 \% ; P>.10 ; 0-\mathrm{sec}$; $\mathrm{Rh}=90 \% ; \mathrm{N}=97.8 \% ; P>.10$; Eacott, pers. comm.). These data are consistent with the findings from the present study. The results reported by Eacott et al. (1994) also are consistent with the present findings in demonstrating a delaydependent deficit following lesions of the PR. For both tasks $\mathrm{A}$ and $\mathrm{A} 1$, the authors reported a significant group $\mathrm{X}$ delay interaction when all the delays were included. The $\mathrm{Rh}$ group was impaired on the MTS task only at the longer delays (15-sec and 30-sec; all Ps <.05; Eacott, pers. comm.), and not at the shorter delays (0 sec and simultaneous matching).

In summary, the results from the present study strengthen the conclusion that the PR and area TE make distinct contributions to visual recognition performance. Together with other recent results from monkeys (Eacott et al. 1994; Buffalo et al. 1999) and from humans (Buffalo et al. 1998a), the present findings do not support the idea that the PR is important for visual perceptual processing and suggest instead that the PR is specialized for the formation of memory and for retaining information across delays. In contrast to the PR, area TE is important for visual information processing.

\section{MATERIALS AND METHODS}

\section{Subjects}

Twelve male cynomolgus monkeys (Macaca fascicularis) were used, all weighing between 3.1 and $5.2 \mathrm{~kg}$ at the start of the study. Five monkeys received bilateral lesions of the PR (PR group), and four received bilateral lesions of inferotemporal cortical area TE (TE group). The remaining three monkeys comprised an unoperated control group ( $\mathrm{N}$ group, $\mathrm{Nx}-\mathrm{Nx}$ ).

\section{Testing History}

Prior to the start of this study, all monkeys had been tested on a number of tasks (Buffalo et al. 1999) and had exactly the same behavioral history. Monkey TE 2 from Buffalo et al. (1999) was not included in the present study. Prior to behavioral testing in the present study, this animal had to be euthanized as a result of gastrointestinal disease. Monkey PR 1 from Buffalo et al. (1999) proved on histological analysis to have significant unilateral damage to area TE on the left side (for detailed description of the damage in this monkey, see Buffalo et al. 1999). This damage was not caused during surgery, but developed during the course of behavioral testing when the left mandibular bone protruded through the craniotomy and compressed the underlying tissue. The damage to area TE was extensive and extended from the pole through the caudal extent of area TE. Because the histological analysis of the lesion was carried out $\sim 5$ years after surgery, it is unclear when, during the course of behavioral testing, the damage to area TE occurred. The behavioral findings in Buffalo et al. (1999), which spanned more than four years of behavioral testing, were presented both with and without monkey PR 1 . The present study reports behavioral results obtained $<1$ year before histological analysis, and it is reasonable to assume that the damage to area TE had already occurred at this time. In any case, we have presented the results both with and without monkey PR 1.

\section{Surgery and Histological Procedures}

The surgical and histological procedures for both the PR and TE lesions have been described in detail previously for these monkeys (Buffalo et al. 1999) and will be described only briefly here.

\section{Surgery}

Monkeys were anesthetized using Isofluorane gas and placed in a specially designed headholder that allowed unobstructed access to the anterior and ventrolateral portions of the temporal lobe. After opening the skull and dura, the pial surface over the intended

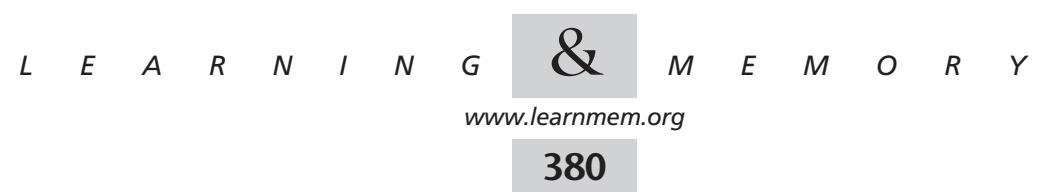


lesions was first cauterized, and the cauterized tissue together with the underlying cortical tissue then was removed by suction until the white matter was visualized.

\section{Location of the Perirhinal Cortex and Area TE}

Our description of the boundaries of the PR follow those of Suzuki and Amaral (1994a). The PR forms a band of cortex along the ventromedial surface of the temporal lobe (Fig. 4). It consists of a smaller, medially situated area 35 and a larger, laterally situated area 36 (Brodmann 1909).

Area TE is located immediately lateral to the PR at most levels and consists of a band of cortex lying primarily on the middle temporal gyrus (Fig. 4). Throughout its rostrocaudal extent, area TE is bordered laterally by superior temporal cortex. Area TEO forms the posterior border of area TE. The description of the area TE - PR border has varied across studies. Based on recent anatomical studies (Suzuki and Amaral 1990; 1994a,b) in M. fascicularis, we have placed the area TE-PR border on the medial bank of the AMTS, $\sim 2 \mathrm{~mm}$ more lateral than in previous studies (Meunier et al. 1993; Eacott et al. 1994).

\section{Intended Perirhinal Cortex and TE Lesions}

For the perirhinal lesions, $\sim 3-4 \mathrm{~mm}$ of cortex lateral to the full rostrocaudal extent of the rhinal sulcus was removed (see Fig. 4). The medial portion of the temporal polar region also was removed.

For the lesions of area TE, the middle temporal gyrus and a portion of the posterior inferior temporal gyrus were removed (see Fig. 4). The intended lesion was bounded laterally by the ventral bank of the superior temporal sulcus (STS).

\section{Histological Verification of Lesions}

The brains were cryoprotected in glycerol solutions and sectioned

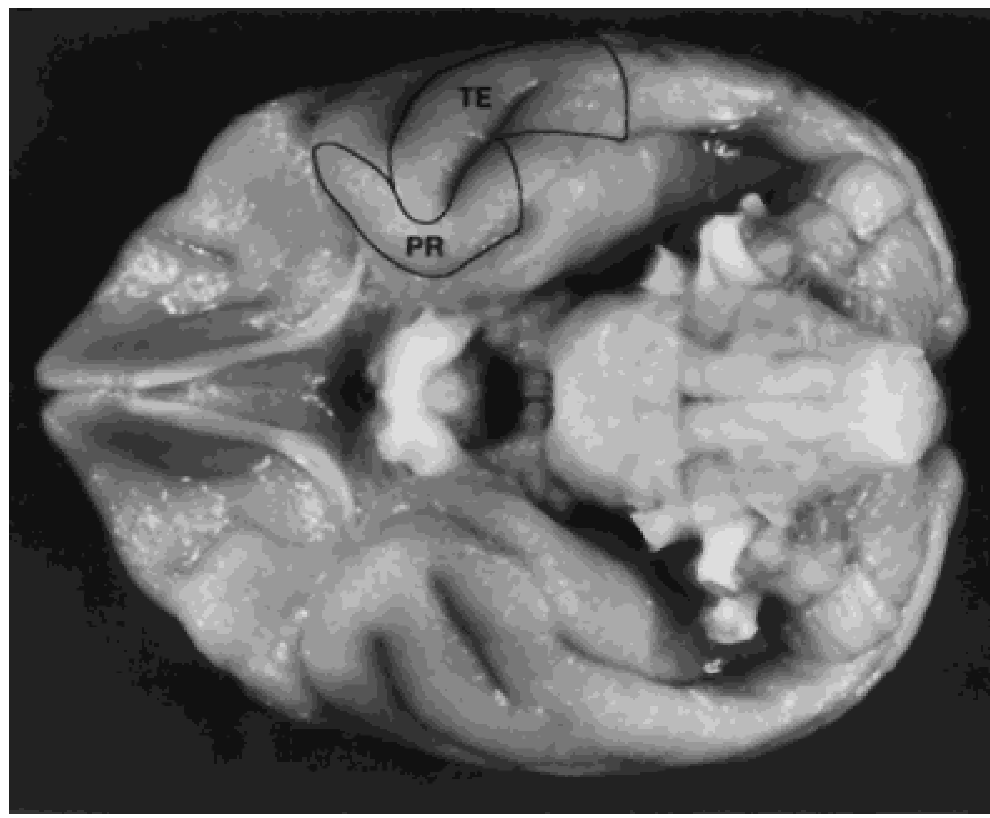

Figure 4 The ventral surface of a macaque monkey brain showing the location of the perirhinal cortex (PR) and inferotemporal cortical area TE (TE). The PR forms a band of cortex along the ventromedial surface of the temporal lobe, lateral to the rhinal sulcus. Area TE is located immediately lateral to the PR and consists of a band of cortex lying primarily on the middle temporal gyrus. See Materials and Methods for a description of the boundaries of the PR and area TE. in the coronal plane at $50 \mu \mathrm{m}$ on a freezing microtome. Every fifth section was mounted and stained with thionin to assess the extent of the lesions. The brains from four monkeys that matched the average weight of each of the two operated groups were used as a histological control group. Thionin-stained sections from the four monkeys in the histological control group, extending from the tips of the temporal poles to the caudal extent of area TE, were scanned into a Macintosh G3 computer at $0.50 \mathrm{~mm}$ intervals. Using National Institutes of Health (NIH) Image software, areal measurements were made of area TE and the PR in each control monkey. The values from these measurements were averaged across all four control monkeys and were used as an estimate of the normal area for the two cortical regions. Similar measurements in each operated monkey yielded an estimate of the area of spared cortex. These values then were used in conjunction with the estimates of the average normal area for PR and area TE to obtain an estimate of the percentage of bilaterally damaged tissue (Buffalo et al. 1999).

\section{Behavioral Testing}

\section{Testing Apparatus}

An IBM-compatible computer operated a touch-sensitive monitor screen (Microtouch Systems) and an automatic pellet dispenser. Visual stimuli were displayed on the monitor and were composed of two superimposed ASCII characters of different shape, size, and color. A contact by the monkey within a $5 \times 6-\mathrm{cm}$ rectangular area centered on the stimulus counted as a response. Correct responses during the choice phase were followed by the delivery of a reward (190-mg banana-flavored monkey pellet; P.J. Noyes) into a small hopper located $5 \mathrm{~cm}$ directly below the center of the monitor.

\section{Automated Visual Delayed Nonmatching to Sample}

Monkeys initially were pretrained in four stages to use the apparatus, as described previously (Alvarez et al. 1994). They first were trained to retrieve rewards from the hopper, then to touch a stimulus when it was presented in the center of the monitor screen, then to touch the stimulus within $60 \mathrm{sec}$ to obtain a reward, and finally to touch a stimulus on the left, center, or right of the screen within $30 \mathrm{sec}$.

\section{Initial Learning}

A sample stimulus was presented in the center of the screen for $20 \mathrm{sec}$ (sample phase). If the monkey touched the stimulus, the screen became blank for 0.5 sec and then the choice phase began. The sample stimulus was not rewarded. If the monkey did not touch the sample, the screen blanked, and the next intertrial interval $(20 \mathrm{sec})$ began. For the choice phase, the sample stimulus and a novel stimulus appeared on the left and right sides of the screen $8.2 \mathrm{~cm}$ from the center. Whether the sample appeared on the left or right side varied according to a predetermined pseudorandom schedule (Gellerman 1933). If the monkey responded correctly, i.e., if it touched the novel stimulus within $20 \mathrm{sec}$, the screen blanked, two banana pellets were delivered, and the intertrial interval began. If the monkey did not respond within $20 \mathrm{sec}$ or responded incorrectly (i.e., if it touched the sample stimulus or if it touched outside the border of the novel stimulus) the screen blanked, no reward was

\section{$\begin{array}{llllllllllllllllllllllllll}L & E & A & R & N & I & N & G & \bigotimes & M & E & M & O & R & Y\end{array}$


delivered, and the intertrial interval began. Monkeys were trained for 40 trials each day until they reached a learning criterion of $90 \%$ correct performance or better within five consecutive days (200 trials). Training was discontinued if a monkey could not reach learning criterion within 3000 trials.

\section{Delays}

After initial learning was completed, monkeys were tested at delays of $1 \mathrm{sec}, 1 \mathrm{~min}$, and $10 \mathrm{~min}$, presented in a mixed order. Specifically, each delay was presented for a block of three days before moving on to the next delay, and the order in which the delays were presented was balanced within and between monkeys. For the 1-sec and 1-min delays, 20 trials were given per day; for the 10-min delay, five trials were given per day. Monkeys received a total of 120 trials at the 1-sec and 1-min delays and a total of 60 trials at the 10-min delay. Monkeys that did not succeed at initial learning (TE 1, TE 3, TE 4, PR 1) were not tested on delays.

\section{ACKNOWLEDGMENTS}

We thank David Amaral for preparation of the PR lesions and Cecelia Manzanares, Scott Hanson, Alisha West, Lawrence Truong, and Michelle $\mathrm{Hu}$ for technical assistance. This research was supported by the Medical Research Service of the Department of Veterans Affairs, NIH Grants MH24600, MH58933, 2T32AG00216 (EAB), and the McDonnell-Pew Center for Cognitive Neuroscience (E.A.B., S.J.R.). E.A.B. is now at the Laboratory of Neuropsychology, National Institute of Mental Health, Bethesda, Maryland. S.J.R. is now at the Department of Psychology, Boston University, Boston, Massachusetts.

The publication costs of this article were defrayed in part by payment of page charges. This article must therefore be hereby marked "advertisement" in accordance with 18 USC section 1734 solely to indicate this fact.

\section{REFERENCES}

Alvarez-Royo, P., Zola-Morgan, S., Squire, L.R. 1992. Impairment of long-term memory and sparing of short-term memory in monkeys with medial temporal lobe lesions: A response to Ringo. Behav. Brain Res. 52:1-5.

. 1994. The animal model of human amnesia: Long-term memory impaired and short-term memory intact. Proc. Natl Acad. Sci. 91:5637-5641.
Brodmann, K. 1909. Vergleichende Lokalisationslehre der Grosshirnrinde. Leipzig: Barth

Buckley, M.J. and Gaffan, D. 1997. Impairment of visual object-discrimination learning after perirhinal cortex ablation. Behav. Neurosci. 111:467-475.

- 1998a. Learning and transfer of object-reward associations and the role of the perirhinal cortex. Behav. Neurosci. 112:15-23.

. 1998b. Perirhinal cortex ablation impairs visual object identification. J. Neurosci. 18:2268-2275.

Buffalo, E.A., Ramus, S.J., Clark, R.E., Teng, E., Squire, L.R., and Zola, S.M. 1999. Dissociation between the effects of damage to perirhinal cortex and area TE. Lrng \& Mem. 6:572-599.

Buffalo, E.A., Reber, P., and Squire, L.R. 1998a. The human perirhinal cortex and visual recognition memory. Hippocampus 8:330-339.

Buffalo, E.A., Stefanacci, L., Squire, L.R., and Zola, S.M. 1998b. A reexamination of the concurrent discrimination learning task: the importance of inferotemporal cortex, area TE. Behav. Neurosci. 112:3-14.

Eacott, M.J., Gaffan, D., and Murray, E.A. 1994. Preserved recognition memory for small sets, and impaired stimulus identification for large sets, following rhinal cortex ablations in monkeys. Euro. J. Neurosci. 6:1466-1478.

Gellerman, L.W. 1933. Chance orders of alternating stimuli in visual discrimination experiments. J. Genetics Psychol. 42:207-208.

Meunier, M., Bachevalier, J., Mishkin, M., and Murray, E.A. 1993. Effects on visual recognition of combined and separate ablations of the entorhinal and perirhinal cortex in rhesus monkeys. J. Neurosci. 13:5418-5432.

Murray, E.A. and Bussey, T.J. 1999. Perceptual-mnemonic functions of the perirhinal cortex. Trends Cog. Sci. 4:142-151.

Overman, W.H., Ormsby, G., and Mishkin, M. 1990 Picture recognition vs. picture discrimination learning in monkeys with medial temporal removals. Exp. Brain Res. 79:18-24.

Suzuki, W.A., Amaral, D.G. 1990. The organization of cortical inputs to the perirhinal (areas 35 and 36) and parahippocampal (areas TH and TF) cortices in the monkey. Soc Neurosci. Abst. 16:53.

- 1994a. Perirhinal and parahippocampal cortices of the macaque monkey: cortical afferents. J. Comp. Neurol. 350:497-533.

- $1994 \mathrm{~b}$. Topographic organization of the reciprocal connections between the monkey entorhinal cortex and the perirhinal and parahippocampal cortices. J. Neurosci. 14:1856-1877.

Received May 20, 2000; accepted in revised form September 18, 2000.

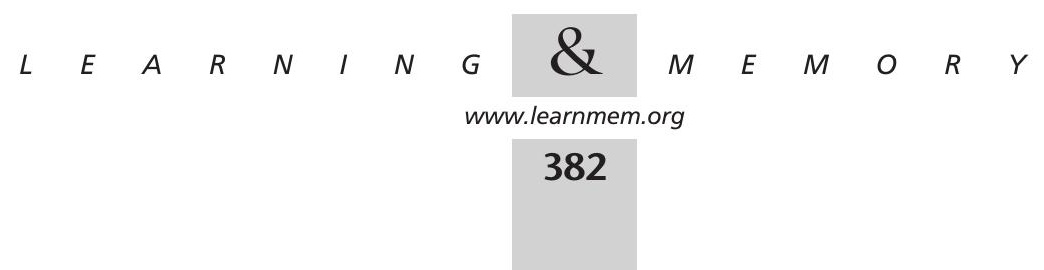




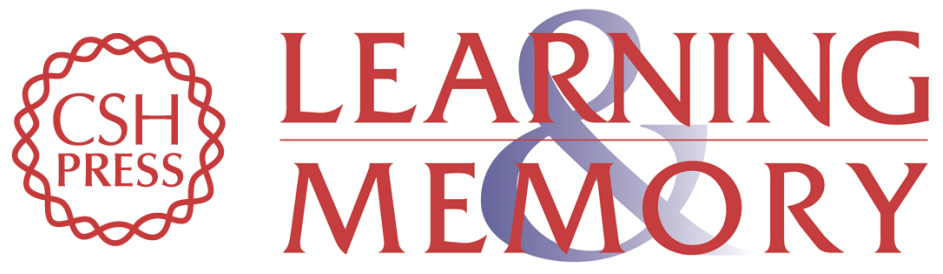

\section{Perception and Recognition Memory in Monkeys Following Lesions of Area TE and Perirhinal Cortex}

Elizabeth A. Buffalo, Seth J. Ramus, Larry R. Squire, et al.

Learn. Mem. 2000, 7:

Access the most recent version at doi:10.1101//m32100

References This article cites 14 articles, 5 of which can be accessed free at: http://learnmem.cshlp.org/content/7/6/375.full.htmI\#ref-list-1

License

Email Alerting Receive free email alerts when new articles cite this article - sign up in the box at the Service top right corner of the article or click here. 